\begin{tabular}{cl} 
Xanthomonadales & \\
Xanthomonas oryzae & 21244927 \\
Xanthomonas fuscans & 294624286 \\
Xanthomas campestris & 78049866 \\
Xanthomonas albilineans & 166714071 \\
Stenotrophomonas sp. SKA14 & 285016900 \\
Stenotrophomonas maltophilia & 254522505 \\
Xylella fastidiosa & 190576418 \\
\hline Actinobacillus minor & 15838553 \\
Aeromonas salmonicida & 257465118 \\
Aggregatibacter aphrophilus & 145301092 \\
Alcanivorax borkumensis & 251793743 \\
Azotobacter vinelandii & 110832869 \\
Cardiobacterium hominis & 226942180 \\
Cellvibrio japonicus & 258545073 \\
Citrobacter koseri & 192359481 \\
Colwellia psychrerythraea & 157149175 \\
Cronobacter turicensis & 71281166 \\
Dickeya dadantii & 260599858 \\
Edwardsiella ictaluri & 242237521 \\
Enhydrobacter aerosaccus & 238917996 \\
Erwinia amylovora & 257453498 \\
Escherichia coli & 292490092 \\
Haemophilus influenzae & 193068544 \\
Hahella chejuensis & 145640588 \\
Idiomarina baltica & 83642934 \\
Klebsiella pneumoniae & 85711017 \\
Mannheimia haemolytica & 206579067 \\
Marinobacter sp. ELB17 & 254362729 \\
Nitrosococcus watsoni & 126668181 \\
Oceanobacter sp. RED65 & 300115242 \\
Pasteurella dagmatis & 94502179 \\
Photobacterium profundum & 260914511 \\
Proteus mirabilis & 54307292 \\
Providencia stuartii & 197286684 \\
Pseudomonas aeruginosa & 183597049 \\
Psychromonas ingrahamii & 296386480 \\
Salmonella enterica & 119947319 \\
Shigella boydii & 56415548 \\
Tolumonas auensis & 187731977 \\
Vibrio furnissii & 237806784 \\
Yersinia ruckeri & 260771056 \\
Ralstonia eutropha H16 & 238754024 \\
Cupriavidus necator N-1 & 113866550 \\
Ralstonia eutropha JMP134 & 338164853 \\
Bordetella pertussis & 73540213 \\
Starkeya novella & 33591304 \\
Rhodobacterales bacterium & 298293954 \\
Oceanicola granulosus & 254465195 \\
Roseovarius nubinhibens & 89070594 \\
& 83952444
\end{tabular}

12

58

GTEELPVKALPGLAQALFDGVLSGLEKRGIAVA RG DAKPLSTPRRLA - . - . - . - - R-F - . - . - - - V - - T

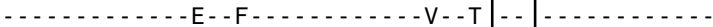

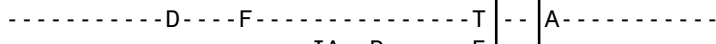

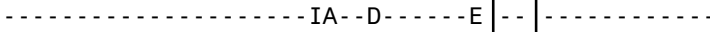
- - - - - - - - - - VD - - R - - V - LD L - - - R - - . -

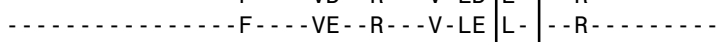
- - - - - N - - N-SR-F-E - - A - . - - V - D H H I E - - S - . . - - . - . - P - - KK - - T - FAEN - EAE - NQA - LSFD KVEWFAA - . . - - A - - - P - - RS - - E - FA - NFKAE - T - ADL - FG - IEWFAS - . - . - . . - . - . - KT - - TSFA-N - EAE - NQA - LTFD KIEWFAA - . . -...-. P - - AS -SA - - T-EFVRQ-DEA-LNHG AVERFAA -... -S - - - P - - KS - - T - FLA - IEK - - AAA - LEYR V-RFYAA - - - V - - - - T - - VTT - - D - GR - LWAQA - ANA - LTHG - IEA - A - . . . -...- P-N-KN-MN - FAESIQAD-TALELSFT SV-AYAA - ... - . - . - P - - RS - - ESFAAN - TAE - DNA - L - HG NVEWFAA - . . - . - . - P-S-KT - - T-FY-NIKGQ-DSHNLSYS - I -WFA - . - F - . - - - P - - RS - - ESFAANFTAE - DAA - LTHG VVSWFAA - ... - - - - - - - RN - - ESFAANFTAE - DTA - LGYQ SVNWFAA - ... - . - . - P - - RS - . - FA - NFRTE - DNA - L - HG - IEWFAA - . . - . - . - P - N-KT - RD - - NNH - TQS - KDAN - RFD TI - SFAA - . . - - - - - P - - RS - - ESFAANLTAE - DAA - L - HG EVSWFAA - ... - - - - - P - - RS - - EFFAANFTAE - DNA - L - HG TVQWFAA - . - . - - - - P - - KT - - TSFA - N - EAE - IQA - LSFD KIEWFAA - - - -A - - - P - - RQ- -E-FAQ-IRQR - -AQLSFS ELTWYAA -... - . - - - P - - KQ - SESFKH - IEE - - N-AEL - FG EI - A-A - . . . - - - - - P - - RS - - ESFAAN - TAE - DNA - L - HG KVEWFAA - . - . - . - . - P - - KK - - T - FAEN - ENE - NQA - LSFE KVEWFAA - . . . . - . - - KQ - SD - FTQ - IV - - QAA - EFG AVE -FAA - ... - . - . - P - - RK - SES - LQELGR - - QAA - LIY - - - L - GYGA - . - . - - - - - PT - - KS - RD - FQK - IETF - QAK - LSF - NSEAFAA - - . - . - . - P - - KK - - T - FAEN - ELE - NQA - LTFD KVEWFAA - . . - . - - P - - RT - - E - FSSNFEAE - KTAALVHQ GIEWFA - . . . - . - . - P - - RS - - ESFATHFTAE - DNAN - THG - VSWFAA - . . - . - - - P - - RS - - ESFAANFTAE - DSAD - - HG EVSWFAA - . - . - . - - - P - - NS - GE - FLS - IEK - - KAA - LGY - A - RFYAA - . - . -...-P-S-RQ - - ESFASN-EAE - N-AELSFD SVRW - AS - ... - . - . - P - - RS - - ESFAANFTAE - DNA - L - HG NVEWFAA - . - . - . - . - P - - RS - - ESFAANFTVE - DNA - L - HG TVQWFAA - . - T - . - . - P - - RK - . - FA- NFTAE - D - A - L - HQ GVQWFAA - . - - - - P- - Q-RT - - E - FAANFTAE - NNA - - HD GVTWYA - . - . - . . - . P - - RS - - ESFAANITAE - DSANLSHG -VSWFAA - ... F - . - . P - - AR - GD - FAQ - LFA - - GE - DLLEP GA TVT - FA - . . . F - . - - P - - GR - GD - FAQ - LFA - - GE - DLLEP GA TVT - FA - . . - . F - - - - P - - AR - GD - FAQ - LFA - - RE - DLLE - DA F - . - - P - - Q QK - G - - FAE - - RAT - ARHHLLAD GC AVE - FA - . . . . FS - - I - ARMQA - A - EN - RKL - TDA - VE - - LLYE G - AFV - . . . . FS - - I - ARMQARA - ED - KKR - TD - -VEA-LTY - G - AA - . - . - T LS - - I - ARMQAKASDD - KKM - TD - - VEA - LTY - S S - AAFA - - - - V FS - - I - ARMQAKA - ED - RQR - TD - - VEA - LTY - G - ASF - . . . - T

\title{
Figure S40
}

Partial sequence alignment of a conserved region of glycyl-tRNA synthetase subunit beta showing a 2 aa insert that is present in all Xanthomonadales. The insert has also been found to be shared by species from $\beta$-Proteobacteria. 